\title{
Nonverbal Communication as a Key Feature of the Nigerian Print Advertising Text
}

\author{
Vareba Anthony Leva, Theophilus Singto Mtodagme, Nwinaene Vivian Peace \\ Department of Mass Communication, Ke Saro-Wiwa Polytechnic, Bori, Nigeria \\ Email address: \\ grace.essoh@yahoo.fr. (V.A. Leva)

\section{To cite this article:} \\ Vareba Anthony Leva, Theophilus Singto Mtodagme, Nwinaene Vivian Peace. Nonverbal Communication as a Key Feature of the Nigerian \\ Print Advertising Text. International Journal of Information and Communication Sciences. Vol. 2, No. 1, 2017, pp. 1-9. \\ doi: $10.11648 /$ j.ijics.20170201.11
}

Received: March 8, 2017; Accepted: March 31, 2017; Published: April 22, 2017

\begin{abstract}
Based on observations, qualitative content analysis and secondary sources, this paper critically examines the importance of nonverbal communication in the Nigerian mass media particularly in print advertising. Hinging principally on two theories (namely semiotics and structuralism theory), the paper argues that despite the fact that they are difficult to systematically be quantified in a mass communication context, nonverbal cues are very important parts to all media producers' efforts to convey emotional, intellectual and informational meanings to audiences. In tandem with this, nonverbal communication cues actually pervade the Nigerian mass media production. The paper demonstrates this fact with illustrations from the Nigerian print advertising. It argues that in the Nigerian print advertising text, nonverbal communication is achieved through specific visual compositions and paralanguage. Print adverts often contain a mix of visuals which are/represent indexical or symbolic signs of positive attributes that advertisers seek to associate with the products or services on promotion. Various forms of paralanguage are equally deployed to suggest the benefits and gratification one may derive from consuming the product or using the services on promotion.
\end{abstract}

Keywords: Nonverbal Cues, Nigerian Mass Media, Subliminal Manipulation, Semiotics, Structuralism, Archetype, Sign

\section{Introduction}

The mass media text has, in most contexts, been a very complex, multidimensional and composite phenomenon. This can egregiously be illustrated by the fact that the print media text - notably magazine, newspaper article and advertising copies - in most cases integrates pure linguistic features (written words), paralinguistic features (typeface, font size [typography] and design) and visual features (cartoons and pictures). Similarly, the audio-visual media text - for instance cinematic productions, pop videos and news casts - has, in most instances, been a combination of verbal, oral and visual elements. Of all the above mentioned constitutive elements, non-verbal cues are indisputably central to the construction and exegesis or decoding of any media text. This is in line with the fact that non-verbal communication is central to effective expression/communication of ideas. Non-verbal communication is thus regarded as powerful and indispensable in most - nay - all efforts to interact with others and transmit mass communication messages. As Vijai insightfully argues, our verbal communication is likely to be ineffective if not accompanied by nonverbal messages. "No matter where we look, nonverbal communication is at the heart of every message conveyed or received whether in face-to-face encounters or over the telephone" [1, p. 19]. According to a great number of studies, the non-verbal cues are more important than verbal elements in any communication. There is even a myth stipulating that nonverbal cues account for $55-65 \%$ of the effectiveness of any communication effort. In view of this, Percy cited by Vijai enthuses that all human communication starts "when words have no meaning" [1]. It begins by the non-linguistic exercises of gazing, rocking, stroking, kissing, and humming. It is through these primordial non-linguistic interactions that a human encoder is connected to his/her interlocutor and the back and forth of communication begins. Hinging on such an observation, nonverbal communication could rightly be regarded as "the core of all languages" [1, p. 20]. In most communication contexts, non-verbal cues contribute to making the message more appealing and interesting to audiences. In effect, nonverbal elements often have over four 
important functions including complementing, regulating, substituting, or emphasizing particular aspects of the verbal message.

In the same line of argument, mass media messages immensely depend on multiple forms of non-verbal communication for their effectiveness. Nonverbal cues basically complement, regulate and put an accent on specific features of mass media messages. This can aptly be illustrated by the fact that media texts often integrate nonverbal cues such as (archetypal) sounds or music to represent specific social worlds and realities. They may, for instance, represent certain regions of the world by deploying types of music that are associated with those worlds or which suggest particular human experiences, through the fact that they integrate suitable/specific archetypal sounds. In line with this, Samba, Rumba or Calypso music is often deployed to represent the South American worlds (in films, documentary and advertising among others), while actualities and sound bites are often used particularly in radio productions to build a mental picture of the news scene in the minds of listeners and communicate cogent realities to audiences. Beach insightfully opines that, the deployment of such archetypal, symbolic or indexical signs - in the form of sound or music - is often based on audience's prior knowledge of certain types of music as being associated with certain types of experiences or worlds [2, p. 23]. Such a deployment equally illustrates media texts constructors' craftiness at using nonverbal communications.

The use of nonverbal cues in the construction of mass media texts can further be illustrated through an intelligent ex-ray of media production in Nigeria. Using a number of theories related to media text interpretation, this paper seeks to examine the place of non-verbal cues in the Nigeria media text or production. It particularly focuses on print advertising by Nigerian organizations. It explores the extent to which myths endorsing the centrality of non-verbal cues in mass communication efforts are pertinent and verifiable. It equally examines the specific aesthetic and communicational values of these nonverbal cues in the Nigerian pint advertising text.

\section{Theoretical Framework}

This paper hinges on the two interrelated theories of semiotics and structuralism. As a theory, Semiotics focuses on the production and interpretation of meaning. It is equally concerned with the cultural and social meaning of signs. A sign is an image, a word, a practice or an object whose meaning principally depends on the relationships between the signifier (the image, word, object, or practice), the signified (the implied meaning), and the referent (what the image, word, object, or practice refers to). Such relation is in many ways dictated or shaped by cultural and societal codes. Black attires and shoved heads as signifiers in some African cultures may have as "signified" bereavement and the morning of a love one. The acts of wearing such black attires and being completely shoved upon the death of loved one or relative may have as referent expression of love and respect for this deceased person [3]. According to Barthes, the meaning of an image, word, object or practice is ideological and cultural [4]. He notes that:

Many semiological systems (objects, gestures, pictorial images) have a substance of expression whose essence is not to signify; often, they are objects of everyday use, used by society in a derivative way, to signify something: clothes are used for protection and food; for nourishment even if they are also used as signs. We propose to call these semiological signs, whose origin is utilitarian and functional, sign-functions. The sign-function bears witness to a double movement, which must be taken apart. In a first stage (this analysis is purely operative and does not imply real temporality) the function becomes pervaded with meaning. This semantization is inevitable: as soon as there is a society, every usage is converted into a sign of itself; the use of a raincoat is to give protection from the rain, but this use cannot be dissociated from the very signs of an atmospheric situation. Since our society produces only standardized, normalized objects, these objects are unavoidably realizations of a model, the speech of a language, the substances of a significant form. [4, p. 37]

Semiotics identifies three typologies for categorizing signs. These include iconic signs, symbolic signs and indexical signs. The iconic sign (for instance photographs or map) resembles that which it describes. There is a physical similarity between an iconic sign and the thing it stands for. A good drawing or picture of a cat - for instance - is very much physically similar to most people's sight of the animal (the cat). An indexical sign acts as a kind of evidence of something; that is, it (the indexical sign) works by association with something connected to it for example sweat is an index for effort; smoke is an index for fire; while crown may, in some circumstances be an index for kingship. In the same line of argument, gun in a cinematic experience may circumstantially be indexical of danger or protection. Symbolic signs on the other hand are visual signs (typically arbitrary in nature) which are linked to referents. In tandem with this, Watson counts letters of the alphabet among examples of this category of signs [5]. He Concedes that "the symbol [symbolic sign] may have no resemblance to what it purports to signify. The letters of the alphabet are symbols. Their meaningfulness as signs exists through common consent and their use is governed by code such as grammatical rules" [5, p. 41]. Other example of symbolic signs may include flags and currency (symbolizing a country); the cross (symbolizing Christianity) and the half moon (symbolizing Islamism) among others.

This paper is equally anchored on the theory of Structuralism. Structuralism is a current which is directly influenced by cultural anthropology, especially that of Claude Lévi-Strauss (who studied myths, kinship systems, rituals). It is centered on the concept of structures - the systems, relations and forms - which make meaning possible in any cultural activity or artifact. From a methodological point of view (aimed at the studying or understanding of "objects" in 
the social sciences), structuralism tends to see "structures" as pre-eminent and its given and directly observable features as mere "effects". Here, structure refers to the mutual relation of the constituent parts of a whole (system) which define its nature, as in "internal structure".

Structuralism stipulates that each element within a cultural system derives its meaning from its relationship to every other element in the system: there are, in effect, no independent meanings, but rather many meanings produced by their difference from other elements in the system. Structuralism therefore hinges on the belief that the individual units of any system have meaning only by virtue of their relations to one another. In line with this, structuralism - in the context of this paper - will be constructed as the intelligent study of (media) text as a whole and the kinds of interrelationships or contrasts that the system builds into itself to give it meaning. Such a system is often constituted of contrasts which are often highlighted by calling attention to their basic oppositional/binary structure. For instance, in a context of newspaper layout, issues such as front versus back page; important versus less important and hard news versus feature stories are relevant here. Also very interesting here might be news versus ads. Though representing technical terminologies/jargons the above mentioned binaries, could be very basic categories of cultural experience (although there could always be an argument about "who's" cultural experience): up/down, culture/nature, male/female.

Structuralism borrows a lot - and definitely follows - from linguistics, particularly that of De Saussure. It is an analytical enterprise dedicated to the systematic elaboration of the rules and constraints that work, similarly to the rules and conventions that govern language, to make meaning generation possible. Early structuralism was therefore distinguished by the use of such Saussurian terminologies as signifier and signified, language and parole; synchronic and diachronic; paradigm and syntagm, which, together with other terms and features of language structures were employed to show how disparate and apparently unorganized phenomena could actually be subtle instances of the same structural patterns and relations, just as all different things that can be said in speech depend on the rulers and constraints of language [6]. Semiotics therefore plays an important role in structuralism. In effect, semiotics considers a number of structuralist principles in its study of sign systems, non-linguistic objects or behaviors. These sign systems and non-linguistic behaviors can be analyzed as if they constituted a language. Semiotics specifically examines the ways non-linguistic objects and behaviors "tell" us something.

Structuralism may therefore imply a degree of nonverbal communication in that, it envisages language as a "sign system" made up of unchanging patterns, rules and codes. Though emerging from a linguistic root, structuralism does not confine communication to the use of linguistic codes. It envisages language (and by extension communication) not just as those things we do when we open our mouths and put some words together in a sentence. Rather, language can be any form of signaling - not just speech or words, but anything that involves communication. For instance, red, yellow and green traffic lights may constitute a form of language or communication. Similarly, national flags, country's currency notes and the like can be forms of (nonlinguistic) communication. Our fancy Nike shoes or Kelvin Klein golden wrist watch that we wouldn't be caught dead without in public may represent key features of the language of "fashion": by sporting those shoes or the golden wrist watch we may be signaling to everyone who stares after us in envy: "I'm stylish, fashionable and loaded".

\section{Nonverbal Cues as Dominant Elements in Communication Contexts}

Before delving into showing the relationship between nonverbal and verbal cues in communicational contexts, it appears expedient to provide a number of definitional clarifications. Nonverbal communication could be defined as behaviors other than words themselves (non-linguistic phenomena) which form a socio-culturally shared coding system. These behaviors are typically used with intent, typically interpreted as intentional ans deployed constantly among members of a socio-cultural community. These behaviors additionally have consensually recognizable interpretations [4]. In interpersonal communication, nonverbal codes are regularly used by communicators to circumstantially telegraph their private thoughts. Most critics tend to limit nonverbal cues to common communicational experiences like body language (kinesics), touching behaviors (haptics), proxemics, paralanguage, oculesics and chronemics among others. However, nonverbal communication should be extended to - or at least be seen in - systematic processes such as subliminal manipulations, structuralism, myth and semiotics. Nonverbal communication is grounded in the deployment of arbitrary signs and symbols which significantly differ from one culture to another. Though the use of nonverbal codes is, for the most cases, arbitrary, it remains possible that a large proportion (of these nonverbal cues) be, to some extent, iconic and/or susceptible to be universally understood. It has for instance been argued that such microexpressions as sadness, anger, fear, disgust, contempt, happiness and surprise are universal. It should be explained here that microexpressions form a specific typology of facial expressions. They are brief, involuntary and hardly faked. They are shown on the face of humans according to the emotions that are being experienced. In her book titled Guide to Reading Microexpressions, Van Edwards illustrates the universal nature of microexpressions when she notes that "people in the US make the same face for sadness as indigenous people in Papua New Guinea who have never seen TV or movies to model. [...] Congenitally blind individuals - those blind since birth, also make the same expressions even though they have never seen other people's faces" [7, p. 4]. 
Nonverbal cues are considered the most important and most powerful variables in any hybrid communication context - that is, in situations where both nonlinguistic and verbal forms of communication are involved. This is not unconnected to the fact that nonverbal cues are believed to be more informative and communicative than verbal communication features. In effect, we tend to rely more on nonverbal signals particularly in situations where verbal and nonverbal messages are clearly concurrent as well as in situations of combined emotional or relational communications [8-13]. For example, when a question is asked in a manner that does not reveal the veritable angle from which the subject (of the question) is approached, the interlocutor or receiver is most often compelled to hinge on nonverbal cues to decode the full meaning of the interrogation. Additionally, nonverbal cues are better indices to detect the credibility of a message and its communicant. For instance the poor use of nonverbal communication by a politician in a context of oration (notably avoidance of eye contact with listeners, monotonous voice, constant/circumstantial stammering and inappropriate hand signals) may be viewed as indexes of incredibility, lack of preparation, or amateurism on the part of the political orator.

Numerous myths thus stipulate that more meaning is produced and generated from nonverbal cues than from verbal ones. Early speculations and imaginations even estimated at $90-93 \%$ the meaning we derived from nonverbal signals in a typical communication context. According to such imaginations, $90-93 \%$ of human communications are nonverbal. In tandem with this, Albert Mehrabian (cited in Blake), conducted several studies on nonverbal communication through which he found that only $7 \%$ of any message is conveyed through linguistic indices (words) while, $38 \%$ of message content is communicated through certain vocal elements, and 55\% through nonverbal elements (facial expressions, gestures and posture among others) [14]. By subtracting the $7 \%$ representing the verbal content, one will deduce the $93 \%$ nonverbal content. The myth of $90-93 \%$ nonverbal content in human communication seems connected to Mehrabian's works and similar studies. However, recent studies have somehow negated such assumptions, reducing the importance of nonverbal communication to percentages ranging from $45-65$. It is important at this juncture to mention that the very dynamic nature of human behaviors (including communication) couple with the limited and often unsystematic nature of some of the methodological approaches used by social scientists to investigate the phenomenon, have rendered the reliable and exact quantification of nonverbal communication an herculean if not impossible phenomenon. However, it remains safe, according to number of observers, to argue that nonverbal communication is powerful and more important than verbal communication behaviors. Such dominance of nonverbal communication will greatly depend on the communicants and the context of communication. As Blake insightfully contends, the fact of the matter is that the exact statistics of verbal versus nonverbal cues in a communication context is irrelevant. "Knowing that communication is specifically $75 \%$ nonverbal or $90 \%$ nonverbal holds no practical applications. The important part is that most communication is nonverbal. In fact, nonverbal behavior is the most crucial aspect of communication" [14, p. 23].

However, there are specific instances in which the reverse will be true - that is, instances in which verbal communication will be richer in meaning than nonverbal cues. Egregious examples are interactions in which information exchange is the unique focus. Schmitz concedes, for instance that, in a context of briefing (at work), verbal communication likely accounts for much more of the meaning generated. However, these kinds of interactions represent just an exception [12]. A key assumption in human communication has been that nonverbal cues are often richer and more revealing in meaning compared to verbal communication, particularly in interpersonal and/or emotional exchanges. It is from this premise that most observers deduce that nonverbal communication is a major feature in mass media production. One will however need enormous and systematic research to aptly test the veracity of this assumption. What is, nevertheless evident is the fact that nonverbal cues are part of the mass media text.

\section{Nonverbal Cues in Mass Media Communication}

The mass media text is most often shaped to reflect or portray human experience and reality; and as such, it is bound to circumstantially incorporate paralinguistic and other forms of nonverbal communication - which form an integral part of human behavior. As pointedly observed by Vinciarelli and Mohammadi, nonverbal cues are appealing from a technological and media point of view because nonverbal cues must necessarily be accessible to our senses (in particular sight and hearing) and this makes them detectable through a wide range of media technologies notably microphones, cameras or other suitable sensors [15]. Media genres such as the filmic text, pop video, comedies and documentaries often offer contexts for the mediation of key aspects of nonverbal communication such as body language, oculecis, and haptics among other. Indeed, in every day fiction, TV news, documentaries, and sports programs, audiences are presented with bodily expressions and other nonverbal cues - by anchors, journalists, and various other participants - as well as characters in film. As Hojbjerg insightfully explains,

The media in which body language is expressed have an influence on how it appears on the big screen and on the flat screen. Mediation is the name of the concept having to do with the way that the media form body language in accordance with the purpose of presentation. Body language is a tool formed in fiction film and television series, which is used to convey meaning in a narrative context. In news and documentaries, body language is mediated in accordance with a standard for presenting 
news and background reports. [16, p. 45]

As noted above, the mediation of body language, microexpressions and other nonverbal cues is done in accordance with specific media codes and genres. Compared to fictional programs, news and other nonfictional programs - which are governed by a number of "rigid" and universalized norms and ethical codes - provide less room for the use of exaggerated body languages. Corroborating this position, Hojbjerg adds that:

It is rather obvious that in news, sports, and documentaries, a certain set of institutionalized contexts determines onscreen body appearance [...] In TV news there is a set of norms as to how to present the news, a set of norms as to how to address the audience, etc. Taken together, these provide a very narrow set of guidelines for managing the anchor's or the speaker's onscreen body performance. Often those guidelines are set by the institution as a kind of codex, but looking at it worldwide, it seems that almost the same basic set of rules applies to news everywhere. The anchors wear makeup, they dress up, and they are rather formal but polite when addressing the audience, in some cases shifting to critically interviewing the guests. Body language in news is determined by media rules. Within this framework, there is a small space for variation of bodily expressions. In most cases, one could say that the body language is, clearly, mediated transparency. In fact, it is a constructed norm for presenting. A classic news flash fits the norm. We are aware of the "constructed" aspect of presenting news, but sometimes body language sticks out as a more exaggerated expression. [16, p. 57]

Media text producers often exploit visual and/or audio channels to communicate non-linguistic messages to audiences. Such nonverbal communication greatly depend on the genre to which a given media production belongs. In print media, still visuals and a number of paralinguistic codes are used while in audio-visual media, archetypal sound special effect sounds (particularly in cinema), and music, accompanied by mediated non-verbal behaviors are often used to produce specific meanings and create a number of effects. In film and comedies precisely, music and sound effects are often used to convey a wide number of unspoken realities. While sound effects are used to convey the ambiance, mood, scope and size, music often assumes a more complex function. As a nonverbal cue in film text, music often communicates emotional, esthetic and even intellectual meanings. It tells the audience how to feel from a sequence of the film to another; it evokes and modulates a specific emotional response in the viewing audiences, to the unfolding narrative, without the audience being aware of it. In his attempt to demonstrate how music and sound effects may function as nonverbal cues in filmmaking, Giamluca notes that:

In film sound, dialogue employs verbal communication, music uses both verbal and non-verbal (but crucially has a recognized 'grammar' of its own in terms of structure and phrasing) whereas sfx would appear to be a purely nonverbal form of communication. Hence, audiences are asked to engage at an intellectual level mostly by dialogue, music occupying a potentially similarly primary position, although in practice often providing emotional engagement for audiences. Sfx on the other hand, lacking a recognized structure, a 'language' as it were, has to be content with the kind of sensual activities traditionally linked to non-verbal forms of communication. In other words, sfx can give you a sense of location, scope, and detail but it cannot engage you at an intellectual level, that being the realm of dialogue and, at times, music. Here again the distinction between verbal and non-verbal, intellectual and sensual, sophisticated and vulgar appear indefensible, unless of course we are bound to understanding cinema solely as a means to convey literal meaning, in which case we could dispense with all sfx and most music, and rely overwhelmingly, if not entirely, on dialogue. [17, p. 23]

The role of nonverbal cues in the meaningful construction of mass media text is therefore more than visible and/or perceptible. Nonverbal communication is facilitated in media text construction by a multitude of tools including subliminal manipulation, structuralism, semiotics and symbolism among others. Structuralism is for instance used as a nonverbal cue in filmic production when directors deploy specific combination and manipulation of shots to convey unspoken realities. The close-ups of faces employed in most soap operas underscores the emphasis most film directors placed on the significance of relationships and emotional conflicts communicated through nonverbal cues. In visual media production (particularly filmmaking), images are often powerful carriers of symbolic and indexical signs which, being intelligently exploited by media producers, tend to subliminally or nonverbally communicate a number of (unspoken) realities. In line with this, Burnett cited by Beach, contends that because images are envisaged as carriers of meaning there is an assumption of fixity which is often equated to a powerful effect of images in all visual experiences. "Effectivity is then used as an argument to explain the referential power of the image" [2, p. 54]. Using Don Segal's film titled Dirty Haris, Burnett further reveals the connotations and symbolic or indexical realities the image of a gun may point to, when placed within the context of a film. He demonstrates how the word "gun" (verbal communication) may significantly defer from the indexical or symbolic used of gun (its image) in a film. He notes that:

Without reference the image would not mean, yet clearly, the object named "gun" is dramatically different from the image named gun. The naming, the classification, is not the same in both instances. For example, the statement that the image of a magnum dominates the film Dirty Harry, by Don Segal, creates more than a simple equation between reference and the language of interpretation. The gun, its 
use, its context, the function which it has in the film, have all been raised and this rather complex discursive field exceeds, transforms, even re-names the object. It is this discursive field which makes the connections between object and image arbitrary. There is no pure moment of the gun as image which escapes its placement and the use to which it has been put. [2, p. 34]

Similarly to filmmakers, both print and audio-visual advertising producers have been exploiting semiotics and structuralism, by technique of associating the product being advertised with relevant visuals or paralinguistic phenomena which symbolize more or less universal virtues or values particularly shared in the culture that receives the advertising message. In line with this, Kilyeni describes advertising copywriting as a complex exercise involving the crafty exploitation of the paralanguage of both pictures and words [18]. With close reference to print adverts placed in women magazines, Kilyeni notes that visuals are deployed to generate connotations and symbolic meanings, intended to be subliminally decoded by readers or viewers of the adverts. In the case of cosmetic products ads for instance, visuals (of female characters) most often have a signifying role. They do not only denote a distinguished personalities or female icon (be she a celebrity or a model), but equally carry connotations such as youth, slimness, health and sensuality which are highly valued in most capitalist cultures. The juxtaposition of pictures carrying such connotations with the product in promotion intrinsically produces another wide range of meanings. As Kilyeni succinctly explains,

By placing together the picture of a woman signifying feminine beauty and that of the cosmetic product, advertisers actually open the way for an indexical transfer from the former onto the latter. In other words, all these positive connotations are carried over onto the advertised product and consequently, women are promised to buy beauty itself together with the product. Images depicting female characters are so widely used in ads primarily because they function as the magic mirror reflection of an embodied perfect self. They invite women to see an improved version of themselves, what they could become if, of course, they used the advertised product. [18, p. 120]

Other nonverbal cues in print advertising include pose (of human characters), setting and props among others are cardinal carriers of meanings, particularly in adverts meant for cosmetic products. As explained by Dyer object props in print advertising are most often signifying and functional. In effect, if in an adverting copy (conceived for milk), milk is presented in a cup, the presence and function of the prop would merely be functional [19]. However if the viewer can associate the cup with some high-quality piece of crockery, the prop's presence in the ad will serve both a functional and a metaphorical function. It may push readers of the advert to transfer connotations of good taste and superior quality onto the milk itself.

In view of the above review on nonverbal cues in mass media text production, it may be safe to argue that images and signs have constituted a site par excellence of nonverbal communication, since "visual communication does not include language codes; it leaves the visual message and the recipient alone" [20, p. 48]. We live in a world in which media producers namely advertisers, filmmakers, graphic artists, image-makers deploy images and signs to intrinsically affect our meaning making process.

\section{Nonverbal Communication in the Nigerian Print Advertising Industry}

The deployment of nonverbal cues in mass media production generally varies in function with the genre, and the socio-cultural codes the readers are to apply in decoding mass media messages. Mass media messages are encoded according to genres and some media codes. In line with this, our analysis in this section will devote attention to the use of nonverbal communication in the Nigerian print advertising industry - which of course may be different from the way nonverbal cues are used in other media. Nigerian advertising copyrighters principally use visuals (iconic and indexical signs) and a wide range of paralinguistic tools as nonverbal cues in the process of designing their messages. These advertising messages sometimes incorporate pictures of human characters representing/symbolizing human beauty, youthfulness, refinement and a number of other positive qualities and values which they indexically or symbolically associate with the products on promotion. This technique is particularly visible in the advertising of cosmetic and beauty products where the pictures of very beautiful, charming and refined women are juxtaposed with the visual (s) of the products being advertised. By so doing the positive characters of the human figures are indexically transferred onto the cosmetic or beauty product. This can be illustrated with images 1 and 2 below.

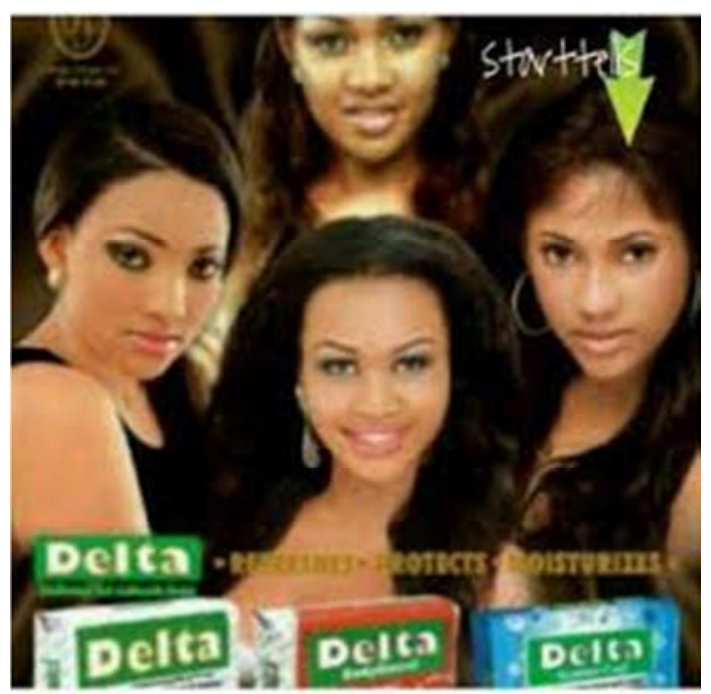

Figure 1. Print Ad of Delta Soap. 


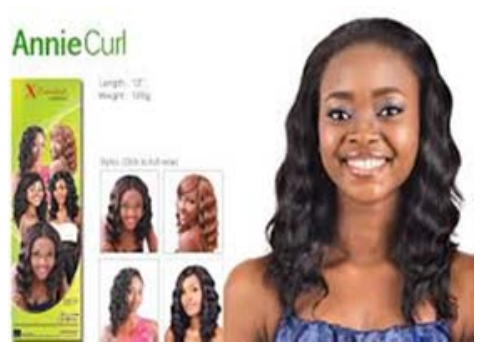

Figure 2. Print Ad of Annie Curl.

In the two above presented images, youthfulness and feminine beauty are intrinsically associated with the products on promotion. The nonverbal message sent by the advertisers, in these two contexts is that, by using the two advertised products (Delta soap and Annie Curl (artificial hair), a female consumer will automatically acquire a beauty and glamour equal to that of the women shown on the advertising copy. The copy shown in Image 1 above particularly emphasizes and celebrates the fair complexion of the women shown on the visual. Even Image 2 indirectly celebrates such a complexion as all the visuals incorporated into the copy are exhibiting women who are fair in complexion. According to popular imaginations and myths prevailing in most Nigerian societies, fair complexion and long and curled hair constitute a mark of female beauty. By juxtaposing female bodies that are moderately or very light in complexion and samples of Delta soap, the copywriters indirectly tells (female) viewers that potential users of the product will ameliorate their pigmentation and ultimately acquire artificial beauty and other physically appealing qualities. Similarly, by sampling visuals of women who are fair in complexion for the advertising of artificial hair Annie Curl copyrighter contextually associates his product with woman beauty.

A wide range of paralanguages are similarly deployed in most print adverts designed by Nigerian advertisers. In effect, the visuals of human characters often deployed in advertising copies, exhibit micro-expressions, haptics, oculecis and kinesics behaviors which complement or re-enforce the verbal contents of the advertising copies. Mobile operators, for instance, recurrently use such mediated nonverbal behaviors (paralanguages) to drive the nail home through visuals. Good illustrations are provided below with the advertising copies of some telephony operators based or operating in Nigeria (see Images 3, 4, 5 and 6 below).

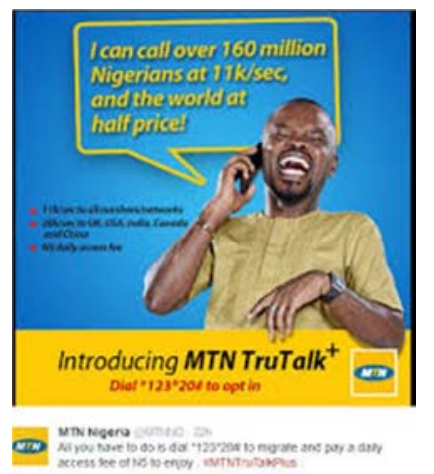

Figure 3. Print Ad of MTN.

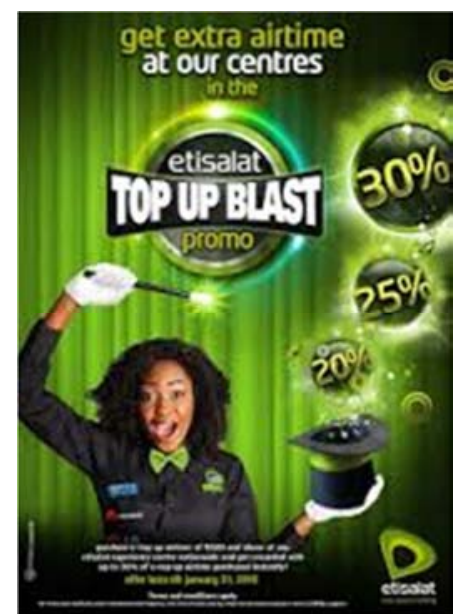

Figure 4. Print Ad of Etisalat.

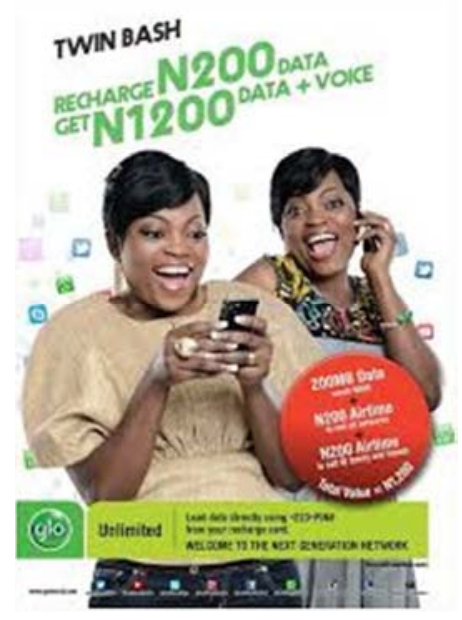

Figure 5. Print Ad of Glo.

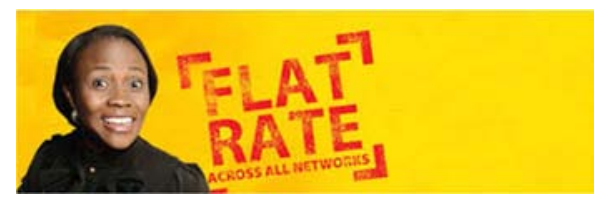

Figure 6. Print Ad of MTN.

In the above advertising copies, mobile operators deploy mediated oculesics and haptics as nonverbal cues to convey both emotional and intellectual meanings. The characters appearing on the copies express monumental surprise and immense joy through their facial expressions (microexpression). Joy and happiness are generally exhibited when (i) the corners of the lips are drawn back and up, (ii) the mouth of the human character is parted and his teeth are exposed, (iii) a wrinkle runs from his/her outer nose to his/her outer lip, (iv) the character's cheeks are raised, (v) his/her lower lid shows wrinkles or is tense and (vi) his/her crows feet near the outside of his or her eyes (Van Edwards, 2013). The implicit message sent through the oculesis and haptics exhibited through these visuals is that the respective mobile operators offer wonderful and unbelievably fair services to their customers. By using their services, the latter (consumers) will be extremely astonished and satisfied. The 
facial expressions of the characters in the visuals indicate that the mobile operators provide more than the expectations of the (potential) consumers. The characters' smiles are inviting as they show a high degree of satisfaction, strength, pleasant surprise and self-sufficiency. Additionally, hand signals and other indexical or symbolic signs are deployed by the copywriters to describe the services offered by these mobile operators. These hand signals often include signs symbolizing OK as shown in Images 7, 8, 9 and 10 below.

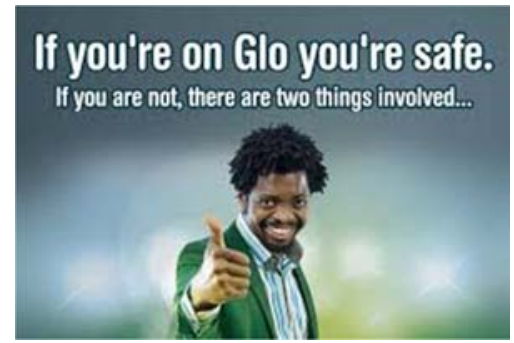

Figure 7. Ad of Glo.

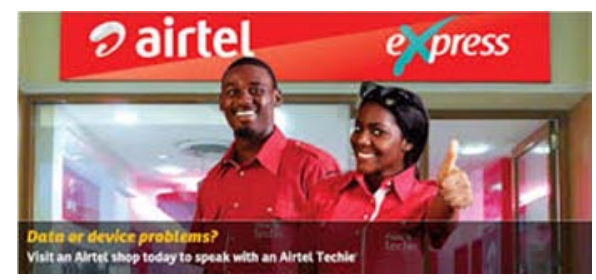

Figure 8. Ad of Airtel.

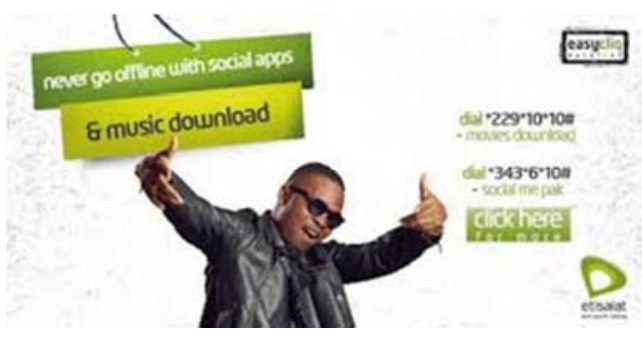

Figure 9. Ad of Etisalat.

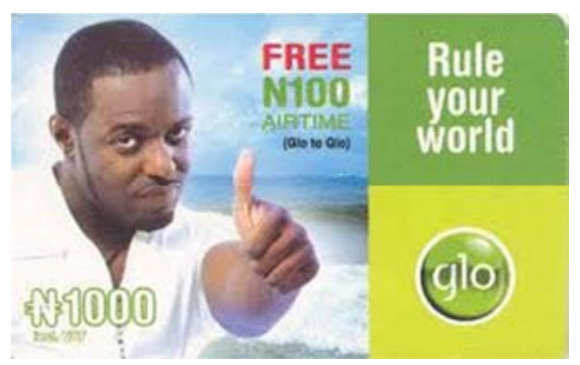

Figure 10. Ad of Glo.

The OK sign exhibited by hand language are invitations addressed to the readers of the copies to similarly endorse the product or services on promotion. They also play the role of nonverbal testimonial. By such hand sign the characters implicitly reveal their experience using the product or the service on promotion. Hand signals are equally deployed to mean uniqueness, victory and power and to indirectly stress that the mobile operators offer services that are clearly distinguished from those of their competitors. This is shown in the images $11,12,13$ and 14 below:

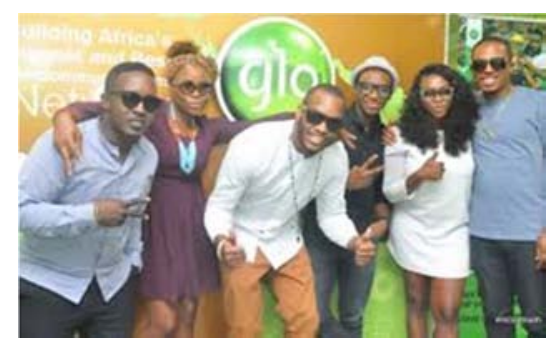

Figure 11. Glo Ad.

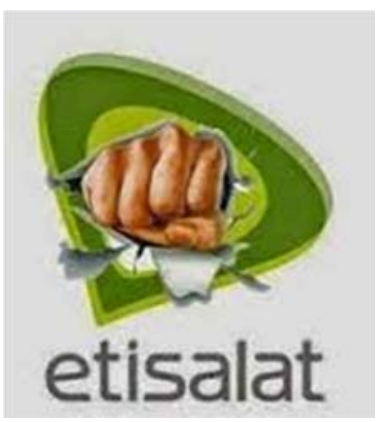

Figure 12. Etisalat Ad.

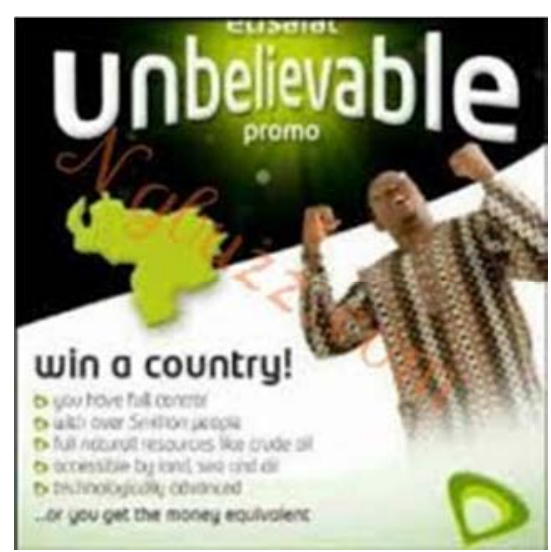

Figure 13. Etisalat Ad.

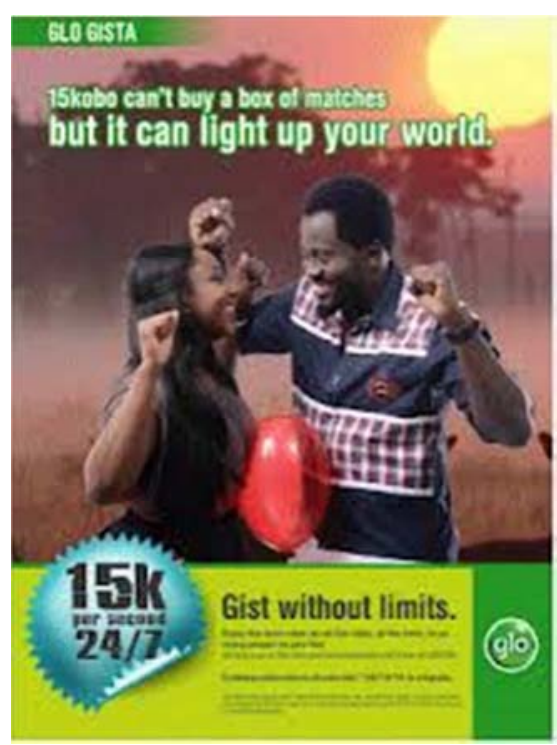

Figure 14. Glo Ad. 
In Image 11 above, characters use a variety of hand language. While some endorse Glo services by brandishing the OK sign (through a thumb sign), others exhibit the Vsign, sign often used by the youth to mean victory or uniqueness. In so doing, they convey the advertising copywriter's message that Glo is unique - there is no other mobile operator which is as functional and practical as Glo. Similarly, Images 12-14 exhibit hand signals symbolizing victory and power (particularly Image 12). One of the probable meanings conveyed here is that users of the advertized services are "winners" or "victors" in that, they will enjoy unique products conceived beyond their imagination/expectations or which other competitors may not provide. The image displayed in Image 12 is more symbolic in that it suggests that Etisalat is "stronger" - and therefore better - than its competitors. Through this image, Etisalat products/services are associated with strength, victory and durability. The visual suggests that the mobile operator's "promises" and selling propositions are durable and more reliable than those of its competitors. It additionally suggests that by using Etisalat, the consumer associates with strength and victory. Though the above illustrations border dominantly on the print advertising of mobile telephony services and cosmetic products, the nonverbal cues discussed in this section are generally observable in the advertising of products through Nigerian print media.

\section{Conclusion}

Nonverbal cues are very important parts of all communication efforts. They actually pervade the mass media. This paper has attempted to show how they are deployed in the Nigerian mass media, particularly in print advertising. It has demonstrated that in Nigerian print advertising, nonverbal communication is achieved through specific visual composition and paralanguage. Print adverts often contain visuals which are indexical or symbolic signs of positive attributes that may be associated with the product on promotion. Various forms of paralanguage are equally deployed to suggest the benefits and gratification one may derive from consuming the product on promotion.

\section{References}

[1] Vijai, G. N. (2009). Non-Verbal Communication Theories". In Littlejohn Stephen W and Karen A. Foss (Eds) Encyclopedia of Communication Theory. London: Sage Publication, 16-31.

[2] Beach, R. (2014). Module 4: "Critical Approaches to Responding to Media Texts". C15472. Teaching Film, Television and Media, New York: Routledge, 56-78.
[3] Endong, F. P. C. (2014). The Effectiveness of Sign-Based Communication in the Advertising/Marketing of Nigerian Products: A User Study. Journal of Harmonized Research in Management, 1 (1), 59-66.

[4] Barthes, R. (1973). Mythologies. London: Grenada/Paladin.

[5] Watson, James. (2003). Media Communication: an Introduction to Theory and Process. New York: Palgrave Macmillan.

[6] O'Sullivan Tim et al. (1996) Key Concepts in Mass Communication. London: Methuen \&Co, Ltd.

[7] Van Edwards, V. (2013). Guide to Reading Microexpression. New York: Science of People LLC.

[8] Branston Gil \& Stafford, Roy (2006). The Media Student's Book. New York: Routledge.

[9] Gun, D. (2015). Subliminal Manipulation, New York: Oxford University Press.

[10] Owen H. (2011). Skilled Interpersonal Interaction: Research, Theory, and Practice, 5th ed. London: Routledge.

[11] Phutela, D. (2015). The Importance of Non-Verbal Communication. IUP Journal of Soft Skills, 9 (4), 15-27.

[12] Schmitz, A. (2012). Non-Verbal Communication. in A Primer on Communication Studies, New York: Routledge, 181-160.

[13] Wales, K. (2001) A Dictionary of Stylistic (2 ${ }^{\text {nd }}$ Edition). Harlow: Longman.

[14] Blake, B. (2014). How Much of Communication is Really Non-Verbal? London: The Nonverbal Group.

[15] Vinciarelli, A. \& Mohammadi, G (2011). Towards a Technology of Nonverbal Communication: A Vocal Behavior in Social and Affective Phenomena. International Journal of Mass Communication, 18 (3), 17-42.

[16] Hojbjerg, L. (2015). The Moving Image: Body Language and Media Context. Kosmorama \# 258, Retrieved August 19, 2016, from http://www.kosmoram.org.

[17] Gianluca, S. (2005). In Defense of Vulgarity: The Place of Sound Effects in Cinema. Film Sound. Learning Space Dedicate to the Art and Analyses of Film Sound Design, New York: Routledge.

[18] Kilyeni, A. (2009). Nonverbal Communication in Print Ads. Professional Communication and Translation, 2 (1-2), 17-24.

[19] Dyer, G. (1999). Advertising as Communication. London: Routledge.

[20] Bullut, T. \& Yardaisik, A. (2005). Visual Semiotics and Interpretation in Television Commercial. AS: Applied Semiotics, 6 (16), 47-53. 